\title{
Is the pulse in atrial fibrillation irregularly irregular?
}

\author{
JOHN M RAWLES, * EDWARD ROWLAND $\dagger$ \\ From the Department of Medicine, University of Aberdeen, ${ }^{\star}$ and the Cardiothoracic Institute, London $\dagger$
}

SUMMARY The belief that there is total irregularity of the pulse in atrial fibrillation has been re-examined. In a computerised analysis of $R-R$ intervals and pulse volumes, $100-500$ (mean 237) consecutive cycles were examined in 74 patients with atrial fibrillation, of whom 36 were on digoxin and 38 were not taking any antiarrhythmic treatment. A Doppler ultrasound technique was used to assess pulse volumes, against which $R-R$ intervals were correlated. Although the sequence of consecutive $R-R$ intervals was random in $52(70 \%)$, patients there was a significant correlation between consecutive intervals in $22(30 \%)$, the correlation coefficient being negative in 11 and positive in 11 . In $43(58 \%$ ) cases the sequence of consecutive pulse volumes was significantly non-random; $34(46 \%)$ showed pulsus alternans, indicated by a negative correlation between consecutive volumes. The proportion of patients with a non-random sequence of $R-R$ intervals or pulse volumes was the same whether or not they were taking digoxin.

Thus patients with atrial fibrillation often have patterns of regularity of the pulse, with the ventricular rhythm being non-random in almost one third and the sequence of pulse volumes being non-random in over a half. Contrary to classic teaching, in many patients with atrial fibrillation the pulse is not irregularly irregular.

Ever since the description of "Pulsus irregularis perpetuus" by Hering in $1903^{1}$ and its identification with auricular fibrillation by Sir Thomas Lewis six years later, ${ }^{2}$ there has been interest in the irregularity and the inequality of the pulse in this condition and the relation between these features. Einthoven and Korteweg clearly demonstrated a positive relation between pulse strength and the preceding $\mathrm{R}-\mathrm{R}$ interval, ${ }^{3}$ and showed also a negative relation between the strength of one pulse and that of its predecessor. They described atrial fibrillation as "pulsus irregularis et inaequalis perpetuus". Lewis later confirmed that the pulse volume in atrial fibrillation was not totally irregular, but that pulsus alternans was commonly found, defining it as "an inverse relation between the strength of a pulse beat and the size of its predecessor." 4 These observations have been generally overlooked and it is widely taught that the pulse in atrial fibrillation is totally irregular in time and volume. ${ }^{56}$

We have examined both the rhythm and the volume of the pulse in patients with atrial fibrillation;

Requests for reprints to Dr John M Rawles, Department of Medicine, University of Aberdeen, Foresterhill, Aberdeen AB9 2ZB.

Accepted for publication 3 February 1986 half were taking digoxin and half were not taking any antiarrhythmic medication. We used statistical techniques to examine each of these characteristics in detail and to determine the relations between them.

\section{Patients and methods}

Seventy four consecutive patients with atrial fibrillation are the subject of this report. There were 34 women and 40 men (mean age 68, range 32-91 years). The aetiology of atrial fibrillation was as follows: idiopathic 33 cases, mitral valve disease 17 , ischaemic heart disease or hypertension or both 19 , chronic obstructive lung disease 2 , pulmonary embolism 2, and thyrotoxicosis 1. Thirty six patients were receiving digoxin; the remainder were not taking any antiarrhythmic medication and had not taken digoxin for at least a week.

In each patient pulse volume was measured by transcutaneous aortovelography, a non-invasive technique in which continuous wave ultrasound was directed at the arch of the aorta from a transducer held in the suprasternal notch. ${ }^{7}$ The Doppler shift frequencies arising from back scattering of ultrasound by aortic blood were subjected to spectral analysis and displayed in real time on a paper trace, 


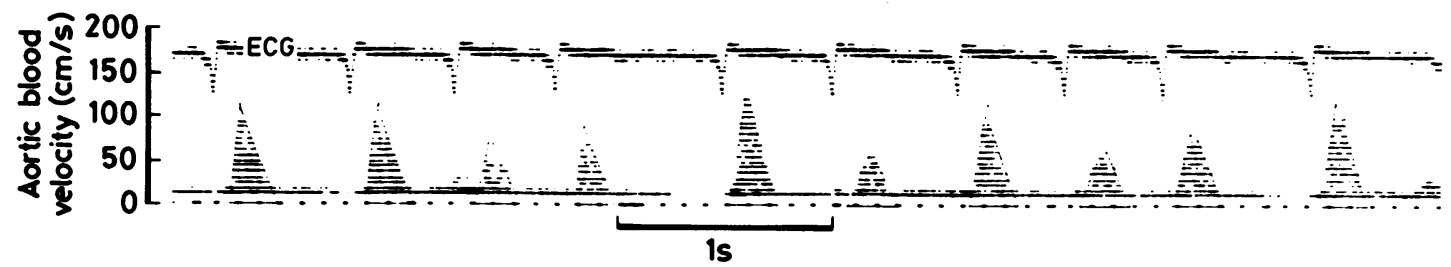

Fig. 1 Record of electrocardiogram and aortic blood velocity obtained by transcutaneous aortovelography in a patient with atrial fibrillation.

which could be directly calibrated as aortic blood velocity.

Figure 1 is a typical trace. The area within the outline of a single velocity-time complex is measured by triangulation to give stroke distance-the distance travelled by aortic mid-stream blood with each beat. We have shown that triangulation gives virtually identical values for stroke distance as those obtained by integration of the area within the envelope. ${ }^{8}$ Elsewhere we have reviewed the evidence that within subjects stroke distance correlates with stroke volume. ${ }^{910}$ A single lead of the electrocardiogram was recorded with the velocity trace at a paper speed of $50 \mathrm{~mm} / \mathrm{s}$. Stroke distances and the corresponding $R-R$ intervals were measured from continuous sequences of up to 500 cardiac cycles, recorded from recumbent patients after at least five minutes' rest. Patients whose electrocardiograms showed evidence of heart block, junctional rhythm, or ventricular extrasystoles were excluded from the study, as were those with evidence of a trend in ventricular rate during the recording - that is all their auto-correlation coefficients were positive. Data from three patients with technically unsatisfactory ultrasound recordings were also rejected.

STATISTICAL METHODS

If the ventricular response in atrial fibrillation is truly irregular then not only should the distribution of $\mathbf{R}-\mathbf{R}$ intervals around the mean be normal, which is usually the case, but their distribution in time should be random. This may be tested by the technique of auto-correlation (Fig. 2). Correlation coefficients are calculated for each $R-R$ interval and its successor (first order auto-correlation coefficient), each interval and its second successor (second order auto-correlation coefficient), third successor (third order auto-correlation coefficient), and so on up to the tenth successor. If the sequence of $\mathbf{R}-\mathbf{R}$ intervals is random and the duration of a given interval has no influence on those that follow then these auto-correlation coefficients will not differ significantly from zero. On the other hand if a given $R-R$ interval has an effect on its successors then one or more of the auto-correlation coefficients will achieve statistical significance that may be negative or positive.

We calculated auto-correlograms for pulse volume, measured as stroke distance, in the same way. Although auto-correlation coefficients up to the tenth order were computed and are illustrated in the figures, higher order coefficients showed no meaningful pattern so only first order coefficients will be further considered in this paper.

To study the relation between $R-R$ intervals and stroke distance we computed a cross-correlogram (Fig. 3). Correlation coefficients are calculated for

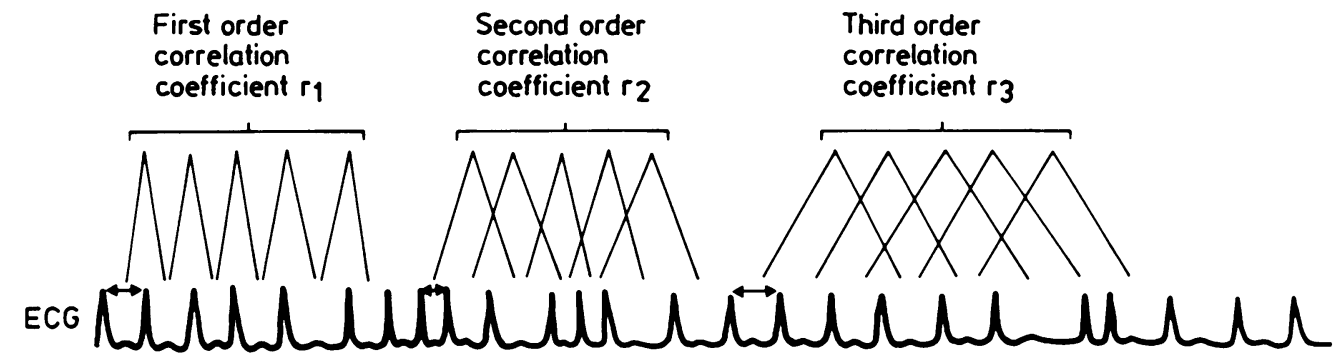

If the sequence of $R-R$ intervals is random then $r_{1}-r_{n}$ are not significant

Fig. 2 The technique of auto-correlation applied to $R-R$ intervals of the electrocardiogram in atrial fibrillation. 


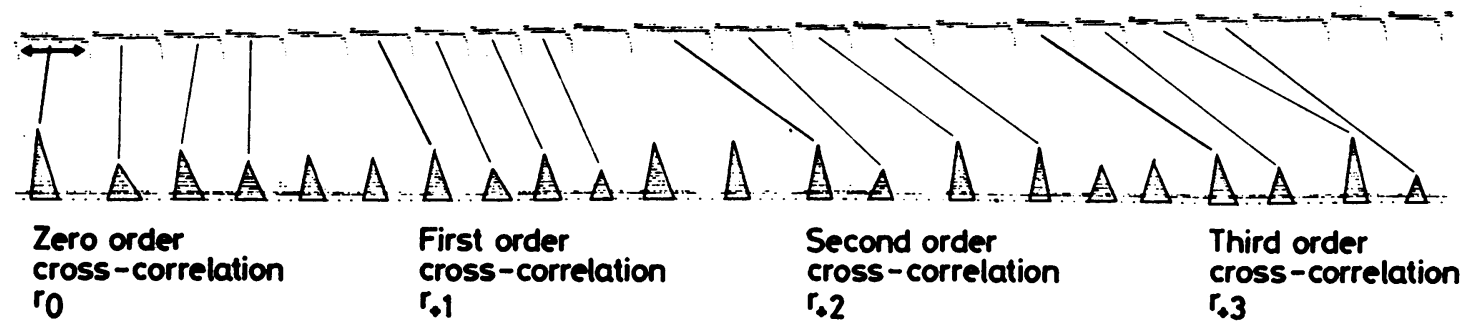

Fig. 3 The technique of cross-correlation applied to $R-R$ intervals and stroke distances.

each $\mathbf{R}-\mathbf{R}$ interval and the stroke distance it includes (zero order), each R-R interval and the stroke distance that follows (first order), and so on up to the tenth cross-correlation. In this manner the influence of an $\mathbf{R}-\mathbf{R}$ interval on subsequent stroke distances can be studied. Cross-correlation coefficients are also calculated between $R-R$ intervals and preceding stroke distances to study the influence of stroke distance on heart rate.

Auto-correlograms for $\mathbf{R}-\mathbf{R}$ intervals and stroke distances, and the cross-correlogram between them were computed from an average of 237 cardiac cycles for each patient (range 100-500). Initially, measurements were made by hand and the statistical analyses were carried out using Minitab on a mainframe computer, but later in the study the recordings were digitised and analysed by a microcomputer.

\section{Results}

The mean ventricular rate was 98 beats $/ \mathrm{min}$, in the whole study group, 87 beats/min in those taking digoxin 87 , and 107 beats/min in those not taking any antiarrhythmic treatment.

Auto-correlation of $R-R$ intervals

Twenty two $(30 \%)$ patients had non-random rhythm, as indicated by a significant first order autocorrelation coefficient for $\mathbf{R}-\mathbf{R}$ intervals (Table 1 ). This was positive in 11 cases and negative in 11 cases. The proportion of patients with a nonrandom rhythm was $32 \%$ in those not taking any antiarrhythmic treatment and $28 \%$ in those taking digoxin (Tables 2 and 3 ).

\section{Auto-correlation of stroke distances}

A non-random sequence of stroke distances was found in $43(58 \%)$ cases, $63 \%$ of those not taking any antiarrhythmic treatment, and $53 \%$ of those taking digoxin (Tables 2 and 3 ). In $34(46 \%)$ cases the auto-correlation coefficient was negative, indicating a tendency for stroke distances to alternate in size, that is to display pulsus alternans.

\section{Relation between randomness of rhythm and of pulse volume sequence}

Of $52(70 \%)$ cases which had a random rhythm, the sequence of stroke distances was random in 22 $(30 \%)$ and non-random in $30(40 \%)$ cases; of 22 $(30 \%)$ cases which had a non-random rhythm, the sequence of stroke distances was random in nine $(12 \%)$ and non-random in $13(18 \%)$ cases. There was no relation between the occurrence of a random rhythm and a random pulse volume sequence $\left(\chi^{2}=0.01, N S\right)$. Tables $1-3$ detail the occurrence of

Table 1 Numbers (\%) of patients with significant $(p<0.05)$ and non-significant first order auto-correlation coefficients (all patients)




Table 2 Numbers ( $\%)$ of patients with significant $(p<0.05)$ and non-significant first order auto-correlation coefficients who were not taking antiarrhythmic treatment

\begin{tabular}{|c|c|c|c|c|c|c|}
\hline & & \multicolumn{5}{|c|}{ First order $r$ for $R-R$ intervals } \\
\hline & & \multirow[t]{2}{*}{ Not significant } & \multicolumn{3}{|c|}{ Significant } & \multirow[t]{2}{*}{ Total } \\
\hline & & & Negative & Positive & Combined & \\
\hline $\begin{array}{c}\text { First order } \\
\quad r \\
\text { for } \\
\text { stroke distance }\end{array}$ & $\begin{array}{l}\text { Not significant } \\
\text { Significant } \\
\text { Negative } \\
\text { Positive } \\
\text { Combined } \\
\text { Total }\end{array}$ & $\begin{array}{lr}9 & \\
& 15 \\
& 2 \\
17 & \\
26(68) & \end{array}$ & $\begin{array}{ll} & 3 \\
3 & 0 \\
5(13) & \end{array}$ & $\begin{array}{l}\quad 4 \\
4 \\
7(18)\end{array}$ & $\begin{array}{ll} & 7 \\
& 0 \\
7 & \\
12(32)\end{array}$ & $\begin{array}{l}14(37) \\
\quad 22(58) \\
2(5) \\
24(63) \\
38(100)\end{array}$ \\
\hline
\end{tabular}

Table 3 Numbers $(\%)$ of patients with significant $(p<0.05)$ and non-significant first order auto-correlation coefficients who were taking digoxin

\begin{tabular}{|c|c|c|c|c|c|c|}
\hline & & \multicolumn{5}{|c|}{ First order $r$ for $R-R$ intervals } \\
\hline & & \multirow[t]{2}{*}{ Not significant } & \multicolumn{3}{|c|}{ Significant } & \multirow[t]{2}{*}{ Total } \\
\hline & & & Negative & Positive & Combined & \\
\hline $\begin{array}{l}\text { First order } \\
\qquad \begin{array}{c}r \\
\text { for }\end{array} \\
\text { stroke distance }\end{array}$ & $\begin{array}{l}\text { Not significant } \\
\text { Significant } \\
\text { Negative } \\
\text { Positive } \\
\text { Combined } \\
\text { Total }\end{array}$ & $\begin{array}{ll}13 & \\
& 6 \\
& 7 \\
13 & \\
26(72) & \end{array}$ & $\begin{array}{ll} & 6 \\
& 0 \\
6 & \\
6(17)\end{array}$ & $\begin{array}{ll} & 0 \\
& 0 \\
0 & \\
4(11) & \end{array}$ & $\begin{array}{cc} & 6 \\
6 & 0 \\
10(28) & \end{array}$ & $\begin{array}{l}17(47) \\
12(33) \\
7(19) \\
19(53) \\
36(100)\end{array}$ \\
\hline
\end{tabular}

the significant and non-significant first order autocorrelation coefficients for $\mathbf{R}-\mathbf{R}$ intervals and stroke distances, subdivided into those not taking any antiarrhythmic treatment and those taking digoxin.

\section{Cross-correlations between stroke distance and $R-R$ intervals}

All patients showed a positive first order crosscorrelation between $\mathbf{R}-\mathbf{R}$ interval and the ensuing stroke distance, and this was significant in all but one case. In 60 out of 74 cases there was a significant cross-correlation between $R-R$ interval and the second succeeding stroke distance, which was negative in 56 cases.

Examples of auto-correlograms and cross-correlograms Figure 4 shows the auto-correlograms and crosscorrelograms from one patient. Correlation coefficients, which may range from -1 to +1 , are plotted against the order of auto-correlation (1 to 10) or of cross-correlation $(-10$ to +10$)$. This patient shows a random sequence of $R-R$ intervals, none of the auto-correlation coefficients being significantly different from zero. The sequence of stroke distances is, however, non-random, the autocorrelogram showing a highly significant negative first order coefficient $(r=-0.28, p<0.001)$ indicating pulsus alternans.

In the cross-correlogram we have chosen to display as positive the order of correlation of a given R-R interval against the stroke distances that follow, and as negative those that precede it. The zero order coefficient is not significant, neither is there any correlation between $R-R$ interval and preceding stroke distances. There is, however, a highly significant positive correlation between $R-R$ interval and the first succeeding stroke distance $(r=0.78, p<0.001)$, and a negative correlation between $R-R$ interval and the second succeeding stroke distance $(r=0.38$, $p<0.001$ ). This patient has a random rhythm but a non-random sequence of stroke distances.

Figure 5 shows the results from a patient with a non-random rhythm and non-random pulse volume sequence. The non-random rhythm is indicated by a significant positive first order auto-correlation coefficient $(r=0.24, p<0.05)$. A positive first order coefficient means that consecutive intervals are more like each other than chance alone would dictate. This patient also demonstrates pulsus alternans $(r=-0.2, \quad p<0.05)$, and significant relations between $R-R$ interval and the two succeeding stroke distances, positive for the first $(r=0.60, p<0.001)$ and negative for the second $(r=-0.28, p<0.05)$, as before. 


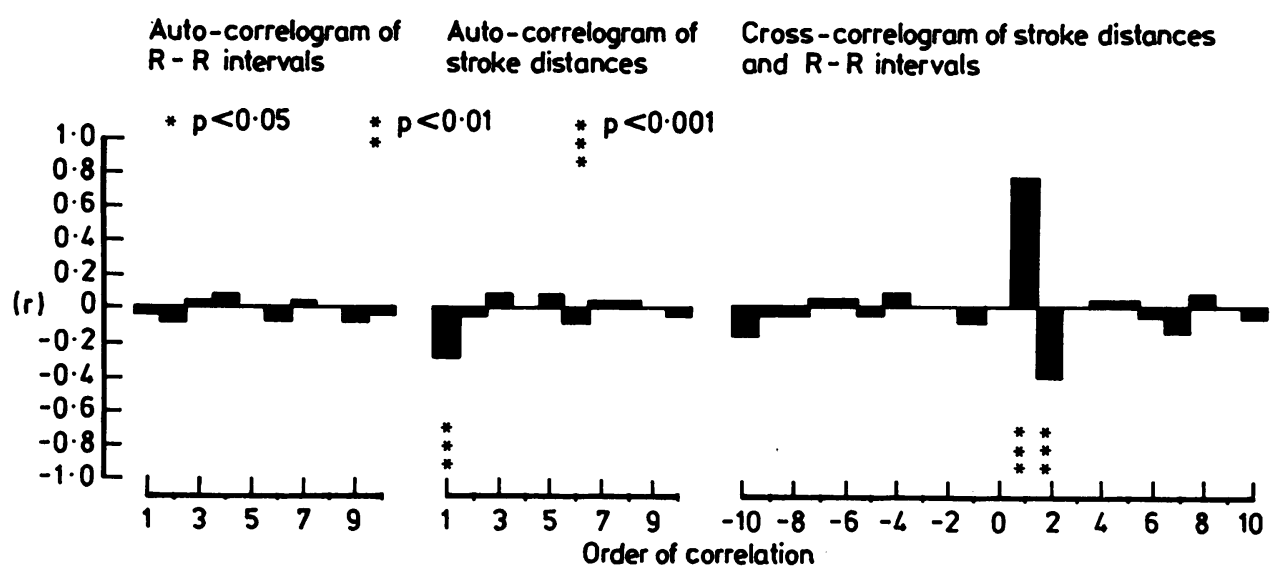

Fig. 4 Auto-correlograms for $R-R$ intervals and stroke distances together with cross-correlogram between them in a patient with random rhythm and non-random sequence of pulse volumes with pulsus alternans.

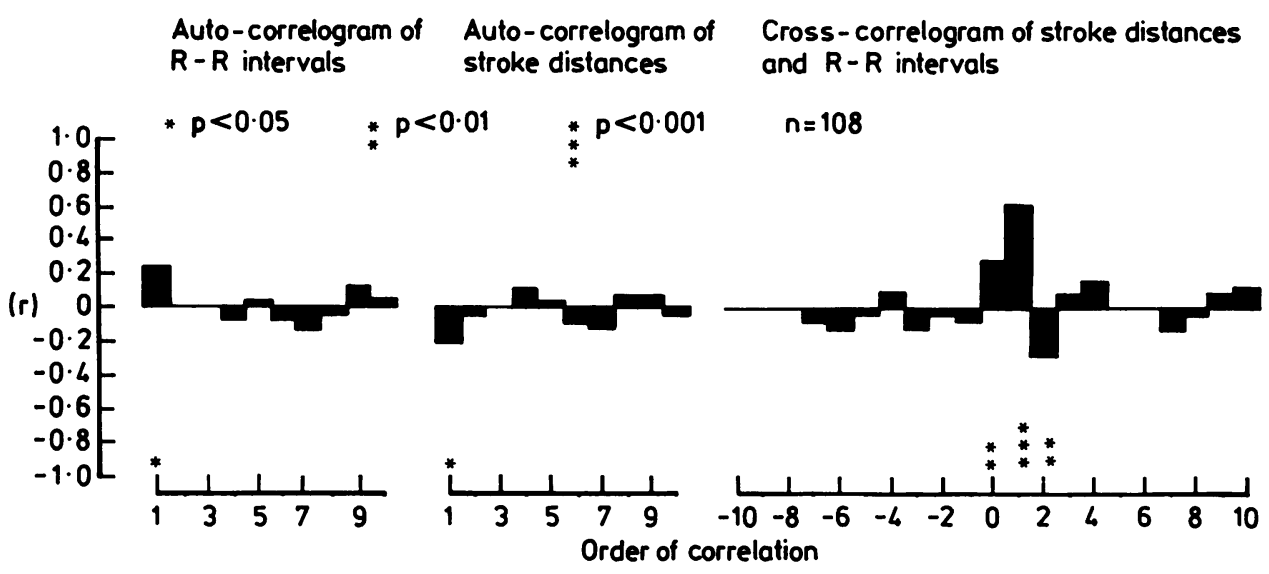

Fig. 5 Auto-correlograms for $R-R$ intervals and stroke distance together with cross-correlogram between them in a patient with non-random rhythm and a positive first order auto-correlation coefficient.

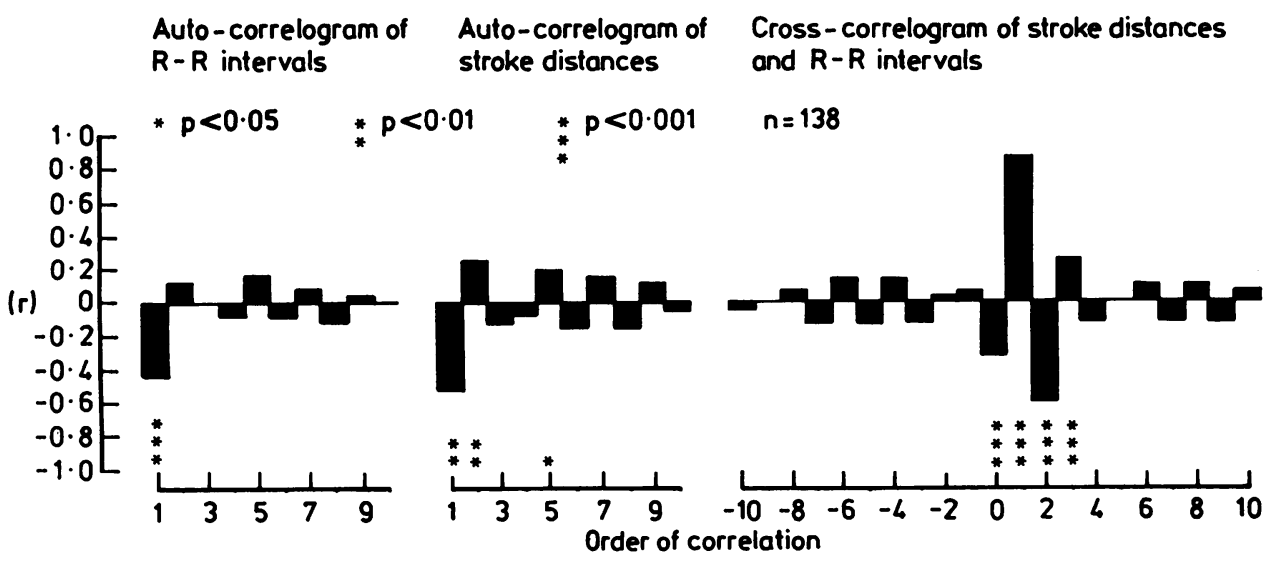

Fig. 6 Auto-correlograms for $R-R$ intervals and stroke distance together with cross-correlogram between them in a patient with non-random rhythm showing alternating cycle lengths and pronounced pulsus alternans. 


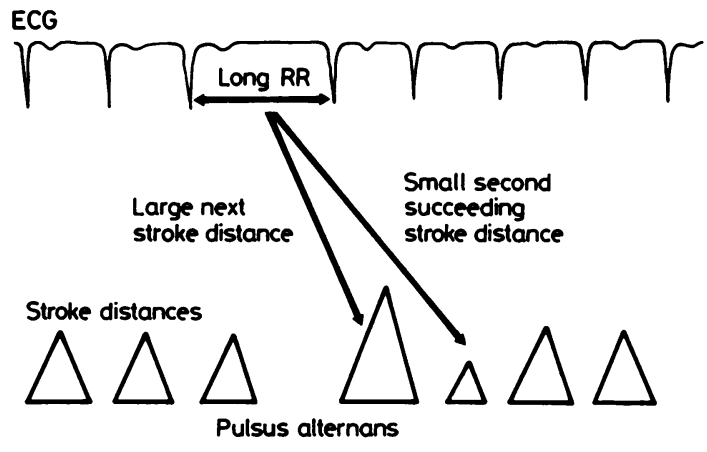

Fig. 7 The mechanism of pulsus alternans.

Figure 6 shows the auto-correlograms and crosscorrelograms from a patient with a non-random rhythm. The first order auto-correlation coefficient for $R-R$ intervals is significant and negative $(r=-0.42, p<0.001)$, indicating that cardiac cycle lengths tend to alternate. Pulsus alternans is also present, indicated by a negative first order coefficient on the auto-correlogram of stroke distance $(r=-0.51, p<0.001)$. There is also a significant positive second order coefficient $(r=0 \cdot 22$, $p<0.01$ ) indicating that stroke distances from every second beat are positively related.

The cross-correlogram shows a very complex pattern of inter-relations, but as before there is a positive correlation between $R-R$ interval and the succeeding stroke distance $(r=0.88, p<0.001)$ and a negative correlation between $R-R$ interval and the second succeeding stroke distance $(r=-0.59$, $\mathrm{p}<0.001$ ).

\section{Pulsus alternans}

Figure 7 shows how a given $R-R$ interval is positively related to the next stroke distance and negatively related to the second succeeding stroke distance. The opposing effect of a given R-R interval on the two succeeding stroke distances results in an inherent tendency to display pulsus alternans when $R-R$ intervals vary in duration, as in atrial fibrillation. In nine out of 11 cases in which $R-R$ intervals alternated there was pulsus alternans (mean $r=-0.37$ ) which was more conspicuous than when it occurred in the presence of a random rhythm (mean $r=-0.27$ ). The presence of pulsus alternans was unrelated to clinical status or the aetiology of the atrial fibrillation, but occurred more frequently $(58 \%)$ in those not taking antiarrhythmic treatment than in those taking digoxin $(33 \%)$. This difference in incidence was not significant $\left(\chi^{2}=0 \cdot 82, \mathrm{NS}\right)$.

\section{Discussion}

It is widely believed that the rhythm of the ventricular response in atrial fibrillation is totally irregular or random, and this was averred in a recent editorial ${ }^{5}$ and article. ${ }^{11}$ In the statistical sense this can never be completely true of atrial fibrillation since there is virtual certainty that for an interval after a given $R$ wave, corresponding to the refractory period of the atrioventricular node, there will not be another $R$ wave, but by the time twice the mean $R-R$ interval has elapsed a second $R$ wave will have occurred. Within these limits $R-R$ intervals show an approximately normal distribution around the mean, though the histogram is often skewed, the median interval being shorter than the mean. Total irregularity is the random sequencing of $R-R$ intervals within this range, with the length of any interval not influencing the length of those that follow. The auto-correlogram is a means of testing whether or not the distribution of R-R intervals in time is random; a series of correlation coefficients is computed between consecutive and higher order events.

In 16 out of 17 patients with atrial fibrillation. Horan and Kistler found no relation between consecutive $R-R$ intervals, ${ }^{12}$ but Goldstein and Barnett found evidence of non-random behaviour in half their records. ${ }^{13}$ They described "chaining", in which series of cardiac cycles with almost equal $R-R$ intervals occur, giving rise to a positive relation between consecutive $\mathbf{R}-\mathbf{R}$ intervals.

Bootsma et al computed auto-correlograms from approximately 2000 beats in 36 patients with atrial fibrillation, 17 of whom were taking digitalis. ${ }^{14}$ These workers maintained that at rest the first and higher order coefficients did not differ from zero. Their published results, however, show that in 12 cases (eight positive and four negative), the first order auto-correlation coefficients exceed the $95 \%$ confidence limit $(\mathbf{0 . 0 6 2}$ for 1000 degrees of freedom (DF)), and in nine cases they exceed the $99 \%$ limit $(0.082, \mathrm{DF}=1000)$. Of 19 patients without treatment, $36 \%$ had a non-random rhythm (three negative and four positive auto-correlation coefficients); this was present in $29 \%$ of patients taking digitalis (one negative and four positive auto-correlation coefficients). In 23 cases the auto-correlograms were repeated after exercise, when 13 positive and one negative first order coefficients were significant $(\mathrm{p}<0.05)$, whereas before exercise three were positive and three were negative. There was a significant $(p<0.05)$ positive shift in the mean value of the first order coefficients with exercise. Bootsma et al observed that an intervention that altered the electrophysiological properties of the atrioventricular node did not affect the random nature of the ventric- 
ular response. From this it was inferred that the random response could not be due to any property of the node but that its explanation must lie elsewhere. Even if the premise of this argument is invalid, as we believe to be the case, the inference is not necessarily altered.

Bootsma et al attributed the random ventricular response that was present in two thirds of their patients to a scaling down by the atrioventricular node of a random sequence of atrial impulses of random amplitude and direction. ${ }^{14} \mathrm{~A}$ similar conclusion was reached by Hashida et al based on a detailed study of a single case at three different ventricular rates. ${ }^{15}$ During atrial fibrillation with a rapid ventricular response the sequence of $R-R$ intervals was random, but at slower ventricular rates, brought about by digitalisation, consecutive $R-R$ intervals became positively correlated, this being attributed to concealed conduction. This differs from the results of Bootsma et al who showed that a positive shift in first order auto-correlation coefficients followed an increase of heart rate with exercise ${ }^{14}$; in five patients who were digitalised there was no change in the coefficients. Moe and Abildskov in experimentally induced atrial fibrillation in the dog showed that stellate ganglionectomy, which reduced the ventricular rate, led to abolition of a positive first order auto-correlation. ${ }^{16}$ Strackee et al, however, pointed out that artificial atrial fibrillation in the dog differs in its rhythmic behaviour from the true form, and so should be used with caution as an experimental model. ${ }^{17}$

In a study of 58 patients with atrial fibrillation, Honzicova et al found a random ventricular response in 51 , but seven had alternation of cycle lengths and a negative first order auto-correlation coefficient. ${ }^{18}$ The random ventricular response of the majority could be simulated by a computer model in which the ventricular response depended on summation of random atrial impulses.

While maintaining that the ventricular rhythm in patients and dogs is always random, Meijler et al demonstrated a non-random rhythm in horses with atrial fibrillation. ${ }^{11}$ Baroceptor responses to blood pressure changes associated with very long $R-R$ intervals were responsible for modulating the ventricular rhythm which would otherwise be random. Meijler et al maintain that this mechanism does not operate in humans because such very long $R-R$ intervals do not occur.

In summary, these studies show that while most cases of atrial fibrillation show a random ventricular response, non-random rhythms with either positive or negative first order auto-correlation coefficients are present in a substantial minority. ${ }^{19}$ There is no convincing electrophysiological explanation to account for both varieties of non-random behaviour or that explains how patients may change from one mode to another. Autonomic tone probably has a major influence on the development of non-random rhythms since pronounced pattern changes accompany changes of mean heart rate.

In our series a non-random ventricular rhythm was present in $22(30 \%)$ out of 74 patients with atrial fibrillation-a result that accords with other published reports. This result is unlikely to be the result of any statistical artefact because we are well aware of the hazards of studying patients with rhythms other than pure atrial fibrillation or of analysing patients whose rhythms exhibit a trend in heart rate, and we did not include such patients. Although we have analysed fewer cardiac cycles than other workers, allowance was made for this by the use of an appropriately increased confidence limit for assessing the significance of any correlation coefficient.

The relation between the rhythm and volume of the pulse in atrial fibrillation has long been of interest. "The absence of a definite and continuing relationship between the strength of a beat and the length of the pause which precedes it", was proposed by Lewis as a criterion for the diagnosis of auricular fibrillation. ${ }^{20}$ Later he modified this view ${ }^{4}$ and credited Einthoven and Korteweg ${ }^{3}$ with the observations that "the strength of the pulse beat is in general related directly to the length of the preceding heart cycle", and "the strength of the beat is also related, inversely, to the strength of the beat which precedes it". This last quotation describes pulsus alternans, although the relation is mathematically negative rather than inverse. Pulse volume in atrial fibrillation is influenced not only by the immediately preceding $R-R$ interval but also by the second and earlier preceding intervals, principally through the force-frequency or Bowditch effect. ${ }^{21} 22$ Using the cross-correlogram the relations between $\mathbf{R}-\mathbf{R}$ intervals and pulse volumes may be displayed, and in our series this confirmed the positive relation between $R-R$ interval and the subsequent stroke distance in every case. Additionally it showed a significant negative relation with the second succeeding stroke distance in $56(76 \%)$ out of 74 cases. The opposing effect of a given $R-R$ interval on the two subsequent stroke volumes readily results in pulsus alternans, which we found in $34(46 \%)$ cases. Alternation of long and short R-R intervals, present in $11(15 \%)$ cases, increases the amplitude of pulsus alternans, as shown by a larger negative correlation coefficient. In atrial fibrillation, unlike sinus rhythm, pulsus alternans does not imply left ventricular dysfunction; we have shown that it is a normal haemodynamic consequence of rapid changes in heart rate. 
The computerised statistical techniques described here demonstrate the frequent occurrence of a nonrandom ventricular response in atrial fibrillation and indicate the complex inter-relations between "pulsus irregularis" and "pulsus inaequalis" during atrial fibrillation. The problem of explaining the non-random element in atrial fibrillation remains, but a possible solution may be found by understanding the mutual interactions between rhythm and haemodynamic function.

We are grateful for the support and encouragement of Dr Dennis Krikler in whose department this work was started during a period of sabbatical leave granted to JR by the University of Aberdeen. A research grant from Grampian Health Board is gratefully acknowledged.

\section{References}

1 Hering HE. Analyse des Pulsus irregularis perpetuus. Prager Medizinische Wochenschrift 1903; 28: 377-81.

2 Lewis T. Auricular fibrillation: a common clinical condition. Br Med $\mathcal{F}$ 1909; ii: 1528.

3 Einthoven W, Korteweg AJ. On the variability of the size of the pulse in cases of atrial fibrillation. Heart 1915; 6: 107-20.

4 Lewis $\mathrm{T}$. The mechanism and graphic registration of the heart beat. London: Shaw and Sons, 1925: 441-7.

5 Meijler FL. Atrial fibrillation: a new look at an old arrhythmia. f Am Coll Cardiol 1983; 2: 391-3.

6 Goldberger E. Textbook of clinical cardiology. St Louis: The CV Mosby Company, 1982: 12.

7 Mowat DHR, Haites NE, Rawles JM. Aortic blood velocity measurement in healthy adults using a simple ultrasound technique. Cardiovasc Res 1983; 17: 75-80.

8 Haites NE, McLennan FM, Mowat DHR, Rawles JM. Assessment of cardiac output by the Doppler ultrasound technique alone. Br Heart $\mathcal{F}$ 1985; 53: 123-9.

9 Rawles JM, Haites NE. Doppler ultrasound mea- surement of cardiac output. Br $\mathcal{F}$ Hosp Med 1984; 31: 292-7.

10 Haites NE, McLennan FM, Mowat DHR, Rawles JM. How far is the cardiac output. Lancet 1984; ii: 1025-7.

11 Meijler FL, Kroneman J, van der Tweel I, Herbscleb JN, Heethar RM, Borst C. Nonrandom ventricular rhythm in horses with atrial fibrillation and its significance for patients. $f \mathrm{Am}$ Coll Cardiol 1984; 4: 316-23.

12 Horan LG, Kistler JC. Study of ventricular response in atrial fibrillation. Circ Res 1961; 9: 305-11.

13 Goldstein RE, Barnett GO. A statistical study of the ventricular irregularity of atrial fibrillation. Comput Biomed Res 1967; 1: 146-61.

14 Bootsma BK, Hoelen AJ, Strackee J, Meijler FL. Analysis of $\mathbf{R}-\mathbf{R}$ intervals in patients with atrial fibrillation at rest and during exercise. Circulation 1970; 41: 783-94.

15 Hashida E, Yoshitani N, Tasaki T. A study on the irregularity of the sequence of $R-R$ intervals in chronic atrial fibrillation in man based on the time series anal-

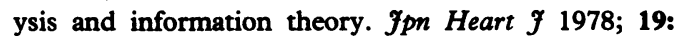
839-51.

16 Moe GK, Abildskov JA. Observations on the ventricular dysrhythmia associated with atrial fibrillation in the dog heart. Circ Res 1964; 14: 447-60.

17 Strackee J, Hoelen AJ, Zimmerman ANE, Meijler FL. Artificial atrial fibrillation in the dog. An artifact? Circ Res 1971; 28: 441-5.

18 Honzicova N, Fiser B, Semrad B. Ventricular function in patients with atrial fibrillation. Cor Vasa 1973; 4: 257-64.

19 Brody DA. Ventricular rate patterns in atrial fibrillation. Circulation 1970; 41: 733-5.

20 Lewis $\mathrm{T}$. The mechanism of the heart beat. London: Shaw and Sons, 1911: 247.

21 Meijler FL, Strackee J, van Capelle FJL, du Perron JC. Computer analysis of the R-R interval-contractility relationship during random stimulation of the isolated heart. Circ Res 1968; 22: 695-702.

22 Gibson DG, Broder G, Sowton E. Effect of varying pulse interval in atrial fibrillation on left ventricular function in man. Br Heart $\mathcal{F}$ 1971; 33: 388-93. 